\title{
Satisfação de Usuários Hipertensos e Profissionais Segundo os Atributos Essenciais da Atenção Primária
}

\author{
Satisfaction of Hypertensive Users and Professionals Based \\ on Primary Care Essential Attributes
}

\section{RESUMO}

Introdução: A Hipertensão Arterial Sistêmica é um dos fatores de risco para as doenças cardiovasculares e a primeira causa de morte no Brasil. Uma forma de avaliar as ações de controle da hipertensão é a satisfação do usuário, pautada em suas experiências no serviço. Todavia, o processo de avaliação deve ser baseado na percepção dos três atores: usuário, profissional e gestor. Objetivo: O objetivo do estudo foi avaliar o desempenho do serviço voltado para o hipertenso tomando como base as dimensões da atenção primária em saúde e comparar os indicadores de satisfação de profissionais e usuários da Estratégia Saúde da Família. Material e Métodos: Estudo transversal, de base populacional realizado com usuários e profissionais da Estratégia Saúde da Família dos municípios de João Pessoa e Campina Grande na Paraíba. Os instrumentos elaborados para o estudo foram baseados no Primary Care Assessment Tool (PCAT) em versões adaptadas e validadas para utilização em hipertensão. Os dados foram analisados utilizando-se o Teste de Mann Whitney, considerando significância estatística para p d" 0,05 . Resultados: Os níveis de satisfação do usuário foram regulares ou baixos, enquanto os indicadores de satisfação dos profissionais foram altos. Conclusão: 0 cuidado não está sendo capaz de atender as necessidades do usuário ou, estas necessidades não estão sendo compreendidas pelos profissionais. O estudo fornece elementos científicos de apoio para a utilização dessa metodologia na avaliação de serviços voltados para o hipertenso.

\section{DESCRITORES}

Avaliação em Saúde. Atenção Primária à Saúde. Hipertensão.

\begin{abstract}
Introduction: Systemic Arterial Hypertension is a risk factor for cardiovascular disease and the leading cause of death in Brazil. User satisfaction based on health care experience can be used to assess the actions focused on the control of hypertension. However, such assessment should embrace the perception of three actors: user, health professional and manager. Objective: This study aims to evaluate the performance of the assistance provided to hypertensive users through primary care dimensions, as well as to compare the indicators of users and professionals of the Family Health Strategy. Materials and Methods: This was a cross-sectional, population-based study performed with users and professionals of the Family Health Strategy in the city ofJoão Pessoa and Campina Grande,Paraíba state, Brazil. An adaptation of the Primary Care Assessment Tool was applied to assess actors' satisfaction with respect to hypertension. Analyses were performed using the Mann Whitney test, considering statistical significance for $\mathrm{p}$ d" 0.05 . Results: Users' satisfaction levels were found to be regular or low, while professional satisfaction levels were found to be high. Conclusion: These findings suggest that the care being provided is not fulfilling users' needs, or that these needs are not being properly understood by professionals. This study is expected to provide scientific evidence supporting the use of this methodology in the assessment of the care provided to hypertensive users.
\end{abstract}

\section{DESCRIPTORS:}

Health Evaluation. Primary Health Care. Hypertension.

Professora das Faculdades ASPER. João Pessoa. Paraíba. Brasil.

2 Professor Associado do Programa de Pós-graduaçao em Modelos de Decisão e Saúde do Departamento de Estatística da Universidade Federal da Paraíba. João Pessoa. Paraíba. Brasil. 
A avaliação em saúde tem o propósito de dar suporte aos processos decisórios no âmbito o sistema de saúde, por meio da identificação de problemas e auxiliando a reorientação das ações e serviços desenvolvidos ${ }^{1}$. Um dos aspectos centrais para a avaliação em saúde está relacionado à qualidade dos serviços prestados. Donabedian considerava a qualidade como um fenômeno complexo do cuidado médico e define a qualidade como um julgamento tanto sobre o componente técnico quanto sobre as relações interpessoais entre o cliente e o profissional ${ }^{2}$.

No processo avaliativo, quando o enfoque é direcionado ao indivíduo, são consideradas as relações que se estabelecem entre prestadores e usuários, bem como as características de ordem técnica referentes ao cuidado em si. Quando o foco de avaliação é o sistema de saúde, são destacados: a acessibilidade, a cobertura e a equidade ${ }^{3}$.

A literatura internacional sustenta que sistemas de saúde organizados que consideram as dimensões essenciais da atenção básica apresentam custos mais baixos, maior qualidade dos serviços, um alto grau de satisfação do usuário, assim como melhor saúde da população, mesmo nas análises controladas por outros determinantes da saúde ${ }^{4}$.

No Brasil, iniciativas de avaliação da Atenção Primária em Saúde (APS) que contribuam para o envolvimento das equipes locais de saúde no processo de melhoria da qualidade dos serviços vêm ganhando destaque ${ }^{1,4-9}$.

A satisfação do paciente tornou-se um dos elementos-chave na avaliação da qualidade em saúde, tornando-se uma meta a ser alcançada pelos serviços, visando o aperfeiçoamento do sistema de serviços de saúde. No Brasil, os estudos sobre satisfação se desenvolveram na década de 1990, por intermédio da participação da comunidade nos processos de planejamento e avaliação ${ }^{10}$. Deste modo, destaca-se a avaliação dos serviços de saúde a partir de uma situação eminente evidenciada no Brasil, que é o crescimento das doenças crônicas, e no caso do estudo a hipertensão arterial, onde seu controle engloba aspectos que envolvam tanto a participação do hipertenso como sua relação com os serviços que são oferecidos e prestados pela APS ${ }^{11}$.

Os estudos que abordam as relações entre os diferentes atores têm criado uma nova perspectiva para a avaliação em saúde. Tais abordagens incluem os atributos essenciais da APS: acesso, porta de entrada, vínculo, elenco de serviços, coordenação, enfoque familiar, orientação para a comunidade e formação profissional ${ }^{4}$. Por conseguinte, o processo de avaliação poderá envolver três tipos de relações interpessoais: usuário $x$ profissional (estudos de percepção dos usuários sobre as práticas, satisfação dos usuários, aceitabilidade, acolhimento, respeito à privacidade $\mathrm{e}$ outros direitos dos cidadãos); profissional x profissional (relações de trabalho e no trabalho) e gestor $X$ profissional (relações sindicais e de gestão) ${ }^{3}$.

A qualidade em saúde tem uma de suas faces voltada para o usuário, com valorização da abordagem humanizada e das relações entre profissionais e pacientes, visando a sua satisfação ${ }^{12}$. Todavia, a satisfação do usuário é apenas uma das vertentes da qualidade. A avaliação do usuário exprime o que o mesmo deseja do serviço. A avaliação do profissional investiga a satisfação das necessidades do profissional e/ou a realização técnica dos procedimentos.

Diante do exposto, a avaliação dos serviços de saúde é direcionada aqui para a relação usuário $x$ profissional tendo como objeto de estudo a hipertensão arterial enquanto foco de interesse da Estratégia de Saúde da Família, no Estado da Paraíba. Para tanto, os dados do presente estudo foram extraídos de uma coorte retrospectiva de hipertensos, ${ }^{13,14}$ cadastrados no Programa HiperDia durante os anos de 2006 e 2007 e que foram acompanhados pelas USFs dos municípios de João Pessoa e Campina Grande na Paraíba em 2009, 2010 e 2011, utilizando como parâmetro de avaliação as dimensões organizacionais da APS. Por conseguinte, teve-se como objetivo principal comparar a satisfação dos usuários e dos profissionais da Estratégia Saúde da Família utilizando os atributos essenciais da APS nos dois maiores municípios da Paraíba em 2010. 


\section{MATERIAL E MÉTODOS}

O presente estudo é um recorte da coorte de hipertensos conduzida no estado da Paraíba ${ }^{13,14}$. Trata-se de um estudo de base populacional, de caráter descritivo e analítico, realizado por amostragem probabilística,com usuários e profissionais das Unidades de Saúde da Família dos municípios de João Pessoa e Campina Grande $-\mathrm{PB}$.

O município de João Pessoa, capital do estado da Paraíba, possuía em 2010 uma população de aproximadamente 723.515 habitantes, segundo dados do IBGE em 2011. O acesso à saúde nos serviços básicos está estruturado em cinco distritos sanitários, com 180 equipes de saúde da família e cinco Unidades Básicas de Saúde (UBS). A cobertura da ESF no município alcançou $90 \%$ em 2011, atendendo acerca de 630 mil pessoas

Campina Grande está localizada no agreste paraibano e se posicionava como o segundo município mais populoso do Estado contabilizando 385.213 habitantes em 2010. O município apresentava um sistema de saúde composto por seis distritos sanitários, 67 Unidades Básicas de Saúde da Família, 92 equipes de saúde da família, cobertura populacional de atendimento na Atenção Primária de 83\% em março de 2011.

A pesquisa com os usuários foi realizada através de uma amostra probabilística e representativa da população de hipertensos cadastrados em 2006/07 nas unidades da ESF nos municípios de João Pessoa e Campina Grande na Paraíba. Os usuários apresentavam idade acima de 19 anos e foram realizadas entrevistas em 2009, 2010 e 2011 com perguntas retrospectivas ao ano anterior, com os mesmos indivíduos, configurando a formação de uma coorte de usuários. A amostra de usuários utilizada neste estudo se refere ao ano de 2010, dos quais 340 hipertensos foram de João Pessoa e 382 de Campina Grande. Depois de contabilizadas as perdas (recusas, mudança de endereço e óbitos), a amostra foi composta por um total de 674 usuários hipertensos. A pesquisa com os profissionais foi realizada em 2010 , com uma amostra representativa e aleatória consistindo de
137 profissionais em João Pessoa e 110 em Campina Grande: médicos, enfermeiros e agentes comunitários de saúde, totalizando 247 profissionais. Aamostra dos usuários e profissionais foi selecionada por processos aleatórios e representativos nas Unidades de Saúde da Família dos municípios no período de novembro de 2009 a março de 2010. O detalhamento do plano de amostragem com a determinação do tamanho da amostra e a extração dos dados fazem parte dos estudos desenvolvidos por Paes ${ }^{13,14}$ que consistiram em avaliar a efetividade no controle da hipertensão arterial sistêmica e associação com fatores de risco dos usuários do Programa de Saúde da Família nos municípios.

Os instrumentos elaborados para o estudo foram baseados no Primary Care Assessment Tool (PCAT) nas versões para usuário e profissional, que foram validados para medir os aspectos críticos da Atenção Primária em Saúde (APS) em países industrializados ${ }^{15}$. O PCAT apresenta parâmetros universais de avaliação por meio das características nucleares da APS: acessibilidade, integralidade, longitudinalidade e coordenação, além de oferecer informações acerca dos aspectos derivativos da Atenção Primária: centralidade na família, orientação comunitária e competência cultural. O PCAT permite a comparação das equipes de Atenção Primária brasileiras com equipes de todo o mundo ${ }^{16}$.

No Brasil, a adaptação e arevalidação foram feitas por meio de um estudo realizado no município de Petrópolis ${ }^{4}$. Todavia, foi necessária nova adequação e validação do instrumento por uma equipe de especialistas para a utilização em indivíduos portadores de hipertensão arterial e para os profissionais avaliados em João Pessoa ${ }^{17}$.

$\mathrm{Na}$ pesquisa foram consideradas as dimensões da atenção primária: Acesso ao Diagnóstico; Acesso ao Tratamento; Adesão/ Vínculo; Elenco de Serviços; Coordenação; Enfoque na Família; Orientação para a Comunidade tanto para os usuários como para os profissionais. As perguntas do instrumento dos profissionais são um espelho das perguntas que constituem o instrumento aplicado aos usuários, mantendo-se, 
portanto, o conteúdo dos elementos avaliados e modificando-se, apenas, a redação da pergunta conforme o ponto de vista: usuário do serviço, profissional de saúde.

A versão do PCAT dos profissionais recolhe informações a respeito de características operacionais e práticas realizadas por profissionais de atenção direta na APS, enquanto a versão aplicada aos usuários coleta informações referentes às experiências do usuário na utilização dos serviços de atenção à saúde ${ }^{15}$.

Cada dimensão foi composta de perguntas com respostas correspondentes a uma escala de possibilidades pré-estabelecidas (Escala tipo Likert), atribuindo-se valores entre um e cinco para as respostas "nunca", "quase nunca", "às vezes", "quase sempre" e "sempre", além das opções "não se aplica" e "não sabe/não respondeu" para captar todas as possibilidades.

Segundo a recomendação do Ministério da Saúde, o indivíduo é considerado acompanhado quando comparece em pelo menos três consultas durante o ano, com registro da pressão arterial no prontuário. E não acompanhado o indivíduo com até duas consultas registradas durante $\mathrm{O}$ ano. $\mathrm{A}$ decisão terapêutica deve ser baseada no risco cardiovascular considerando-se a presença de fatores de risco, lesão em órgão-alvo e/ou doença cardiovascular estabelecida, e não apenas no nível da PA. Todavia, os valores aceitos como normotensão ou hipertensão controlada, medidos em consultório, devem ser abaixo de $140 / 90 \mathrm{mmHg}^{18}$.

O perfil dos hipertensos foi investigado no instrumento por meio das variáveis: sexo, faixa etária, tipo de usuário, etnia, escolaridade, situação conjugal e controle pressórico. Para os profissionais investigou-se a função, a titulação e o tempo de trabalho na função.

Foi calculado um índice composto para cada dimensão por meio da média dos escores dos indicadores (variando entre um e cinco) nas duas versões. Após a construção dos índices compostos para analisar o grau de satisfação dos hipertensos, a escala dos valores médios foi reclassificada como: valores abaixo de 3 (insatisfatório); entre 3 e 4(regular); acima de 4 (satisfatório).
Averiguações preliminares usando o teste de Kolmogorov-Smirnov para constatar a normalidade dos dados não se confirmou, o que justificou a escolha do teste não paramétrico $U$ de Mann-Whitney para a comparação das médias dos índices compostos (que medem o grau de satisfação das respostas) para o ano de 2010 , atribuindo-se um nível de significância de $5 \%$. As comparações foram realizadas entre Usuários e Profissionais do mesmo município, segundo as dimensões da atenção primária e para as mesmas dimensões entre os municípios.

Para a construção do banco de dados foram utilizados os softwares Microsoft Office Access ${ }^{\circledR}$, Microsoft Office Excel $\AA^{\circledR}$ e as análises estatísticas no IBM SPSS Inc. PASW Statistics versão 20.0. A pesquisa foi apreciada e aprovada pelo Comitê de Ética e Pesquisa do Hospital Lauro Wanderley da Universidade Federal da Paraíba, protocolo n ${ }^{\circ} 0101$ de 29/04/2009.

\section{RESULTADOS}

Na Tabela 1 é apresentada a distribuição geral de todos os hipertensos participantes do estudo por tipo de usuário, segundo variáveis sociodemográficas e pressão arterial para o ano de 2010, em João Pessoa e Campina Grande.

A faixa etária predominante foi de idosos (acima de 60 anos) tanto em João Pessoa, quanto em Campina Grande. Com relação à etnia, o percentual de não brancos foi predominante em ambos os municípios. Dentre os participantes, observou-se um baixo nível de escolaridade (analfabetos e com educação básica) nos dois municípios.

Com relação à situação conjugal, foi observado um perfil semelhante nos dois municípios: a maioria dos indivíduos morava com o companheiro, filhos e/ou outros,e um baixo percentual de indivíduos que moravam sozinhos.

Para o presente estudo, a avaliação do serviço de saúde foi feita indiretamente por meio da avaliação do controle pressórico dos indivíduos cadastrados no HiperDia. Os usuários foram 
avaliados quanto ao controle ou não da pressão arterial.

Foi encontrado um alto percentual de indivíduos com níveis pressóricos não controlados em João Pessoa e Campina Grande.

A Tabela 2 mostra a distribuição dos profissionais quanto ao tempo de atuação na função e a titulação para os municípios de João Pessoa e Campina Grande. Observou-se um elevado percentual de profissionais com pouco tempo na função (até 2 anos) para o município de João Pessoa.

Para Campina Grande observou-se um considerável percentual de profissionais com 4 a 12 anos na função. Quanto à titulação, observou- se um elevado percentual de especialistas/ pósgraduados entre médicos e enfermeiros.

A Tabela 3 mostra os valores descritivos dos indicadores obtidos nas sete dimensões presentes para os dois atores participantes do estudo em cada município. As respostas em grau de satisfação variavam segundo a Escala tipo Likert de um mínimo 0 (zero) a um máximo 5 (cinco).

As maiores médias foram atribuídas pelos profissionais de ambos os municípios, com destaque para a dimensão Adesão/Vínculo que apresentou a média mais elevada. Com relação aos usuários, as médias mais elevadas foram atribuídas pelos hipertensos de João Pessoa.

A Tabela 4 apresenta o resultado da

\begin{tabular}{|c|c|c|c|c|c|c|c|c|c|c|c|c|}
\hline \multirow{3}{*}{ Variáveis } & \multicolumn{6}{|c|}{ João Pessoa } & \multicolumn{6}{|c|}{ Campina Grande } \\
\hline & \multicolumn{2}{|c|}{ Acompanhado } & \multicolumn{2}{|c|}{ Não Acomp } & \multicolumn{2}{|c|}{ Total } & \multicolumn{2}{|c|}{ Acompanhado } & \multicolumn{2}{|c|}{$\begin{array}{c}\text { Não } \\
\text { Acomp }\end{array}$} & \multicolumn{2}{|c|}{ Total } \\
\hline & $\begin{array}{c}n= \\
93\end{array}$ & $\%$ & $\begin{array}{l}n= \\
221\end{array}$ & $\%$ & $\begin{array}{l}n= \\
314\end{array}$ & $\%$ & $\begin{array}{l}n= \\
182\end{array}$ & $\%$ & $\begin{array}{l}n= \\
172\end{array}$ & $\%$ & $\begin{array}{l}n= \\
354\end{array}$ & $\%$ \\
\hline \multicolumn{13}{|l|}{ Sexo } \\
\hline Feminino & 70 & 75,3 & 159 & 71,9 & 229 & 72,9 & 151 & 83 & 129 & 75 & 280 & 79,1 \\
\hline Masculino & 23 & 24,7 & 62 & 28,1 & 85 & 27,1 & 31 & 17 & 43 & 25 & 74 & 20,9 \\
\hline \multicolumn{13}{|l|}{ Faixa Etária } \\
\hline Adulto (20 a 59 anos) & 39 & 41,9 & 119 & 53,8 & 158 & 50,3 & 83 & 45,6 & 79 & 45,9 & 162 & 45,8 \\
\hline Idoso (60 anos acima) & 54 & 58,1 & 102 & 46,2 & 156 & 49,7 & 99 & 54,4 & 93 & 54,1 & 192 & 54,2 \\
\hline \multicolumn{13}{|l|}{ Etnia } \\
\hline Branco & 32 & 34,4 & 77 & 34,8 & 109 & 34,7 & 76 & 41,8 & 76 & 44,2 & 152 & 42,9 \\
\hline Não branco & 52 & 55,9 & 135 & 61,1 & 187 & 59,6 & 106 & 58,2 & 96 & 55,8 & 202 & 57,1 \\
\hline Sem informações & 9 & 9,7 & 9 & 4,1 & 18 & 5,7 & 0 & & 0 & & & \\
\hline \multicolumn{13}{|l|}{ Escolaridade } \\
\hline Analfabeto & 15 & 16,1 & 40 & 18,1 & 55 & 17,5 & 30 & 16,5 & 29 & 16,9 & 59 & 16,7 \\
\hline Educação Básica & 67 & 72 & 164 & 74,2 & 231 & 73,6 & 151 & 83 & 138 & 80,2 & 289 & 81,6 \\
\hline Educação Superior & 4 & 4,3 & 9 & 4,1 & 13 & 4,1 & 1 & 0,5 & 5 & 2,9 & 6 & 1,7 \\
\hline Sem informações & 7 & 7,5 & 8 & 3,6 & 15 & 4,8 & 0 & & 0 & & 0 & \\
\hline \multicolumn{13}{|l|}{ Situação conjugal } \\
\hline $\begin{array}{l}\text { Convive comp, filho } \\
\text { e/ou out }\end{array}$ & 82 & 88,2 & 204 & 92,3 & 286 & 91,1 & 168 & 92,3 & 162 & 94,2 & 330 & 93,2 \\
\hline Vive só & 4 & 4,3 & 6 & 2,7 & 10 & 3,2 & 14 & 7,7 & 9 & 5,2 & 23 & 6,5 \\
\hline Sem informações & 7 & 7,5 & 11 & 5 & 18 & 5,7 & 0 & & 1 & 0,6 & 1 & 0,3 \\
\hline \multicolumn{13}{|l|}{ Pressão Arterial } \\
\hline Controlada & 29 & 31,2 & 66 & 29,9 & 95 & 30,3 & 81 & 44,5 & 66 & 38,4 & 147 & 41,5 \\
\hline Não Controlada & 56 & 60,2 & 149 & 67,4 & 205 & 65,3 & 93 & 51,1 & 104 & 60,5 & 197 & 55,6 \\
\hline Sem informações & 8 & 8,6 & 6 & 2,7 & 14 & 4,5 & 8 & 4,4 & 2 & 1,2 & 10 & 2,8 \\
\hline
\end{tabular}




\begin{tabular}{|c|c|c|c|c|}
\hline \multicolumn{5}{|c|}{$\begin{array}{l}\text { Tabela 2: Distribuição absoluta e rel ativa dos profissionais } \\
\text { segundo o tempo na função e titulação para João Pessoa e } \\
\text { Campina Grande, } 2010 \text {. }\end{array}$} \\
\hline & \multicolumn{2}{|c|}{$\begin{array}{c}\text { João Pessoa } \\
n=137\end{array}$} & \multicolumn{2}{|c|}{$\begin{array}{c}\text { Campina Grande } \\
\qquad n=110\end{array}$} \\
\hline Tempo na função & $\mathbf{n}$ & $\%$ & $\mathbf{n}$ & $\%$ \\
\hline Até 2 anos & 94 & 68.1 & 40 & 36.4 \\
\hline 2 a 4 anos & 10 & 7.2 & 20 & 18.2 \\
\hline 4 a 12 anos & 33 & 33.0 & 42 & 38.2 \\
\hline Acima de 12 & 0 & - & 8 & 7,3 \\
\hline Não informou & 1 & 0,7 & 0 & - \\
\hline \multicolumn{5}{|l|}{ Titulação } \\
\hline Médicos & 29 & 21.2 & 26 & 23.6 \\
\hline Graduação & 5 & 17.9 & 4 & 15.4 \\
\hline Especialista/Pós qraduado & 17 & 60.7 & 22 & 84.6 \\
\hline Não informou & 6 & 21.4 & 0 & - \\
\hline Enfermeiros & 36 & 26,3 & 28 & 25.5 \\
\hline Graduação & 8 & 22,2 & 5 & 17,9 \\
\hline Especialista/Pós qraduado & 24 & 66.7 & 23 & 82.1 \\
\hline Não informou & 4 & 11.1 & 0 & - \\
\hline ACS & 72 & 52,6 & 56 & 50.9 \\
\hline $2^{\circ}$ Grau completo & 43 & 72.9 & 1 & 1,8 \\
\hline $2^{\circ}$ Grau incompleto & 1 & 1.7 & 0 & - \\
\hline Superior completo & 3 & 5.1 & 3 & 5.4 \\
\hline Superior incompleto & 11 & 18.6 & 6 & 10,7 \\
\hline Especialista & 1 & 1.7 & 0 & - \\
\hline Não informou & 0 & - & 46 & 82.1 \\
\hline
\end{tabular}

\begin{tabular}{|l|c|c|c|c|c|c|c|c|c|c|c|c|c|}
\hline \multicolumn{1}{|c|}{ Tabela 3: Estatística descritiva para Usuários e Profissionais, segundo o grau de satisfação por } \\
\hline
\end{tabular}




\begin{tabular}{|c|c|c|c|c|}
\hline Dimensões & Usuário JP/CG & Prof JP/CG & $\begin{array}{l}\text { Usuário/Prof } \\
\text { JP }\end{array}$ & Usuário/Prof CG \\
\hline Acesso ao Diagnóstico & $0,000^{*}$ & 0,008 & $0.000^{*}$ & 0.404 \\
\hline Acesso ao Tratamento & $0,001^{*}$ & $0,004^{*}$ & $0,000^{*}$ & $0,000^{*}$ \\
\hline Adesão/Vínculo & 0,577 & 0,450 & $0,000^{*}$ & $0.000^{*}$ \\
\hline Elenco de Serviços & $0,000^{*}$ & 0,631 & $0,000^{*}$ & 0,075 \\
\hline Coordenação & $0,000^{*}$ & $0,000^{*}$ & 0,053 & $0,000^{*}$ \\
\hline Enfoque na Família & 0,908 & 0,900 & $0,000^{*}$ & $0.000^{*}$ \\
\hline Orientação Comunidade & $0,000^{*}$ & $0,001^{*}$ & $0,000^{*}$ & $0,000^{*}$ \\
\hline
\end{tabular}

comparação dos graus de satisfação dos usuários e profissionais com a mesma dimensão, nos dois municípios utilizando o teste $\mathrm{U}$ de Mann Whitney. Ao comparar a satisfação dos atores entre os dois municípios observou-se uma maior diferença na resposta dos usuários. Ao comparar as categorias (usuários e profissionais) em cada município observou-se maior número de dimensões apresentando diferenças para o município de João Pessoa.

\section{DISCUSSÃO}

A avaliação em saúde pautada na opinião de usuários e profissionais de um serviço associase ao conceito de satisfação que, por sua vez, pode traduzir a qualidade do atendimento prestado. Diversos modelos teóricos sobre o conceito de satisfação receberam críticas devido a sua complexidade e ausência de rigor metodológico. Em muitos deles, avaliava-se a satisfação subjetivamente a partir das expectativas do usuário, ressaltando-se que o termo "satisfação" é bastante complexo e que nem sempre é compreendido pelo usuário. ${ }^{10}$

Partindo do pressuposto de que o processo avaliativo da qualidade do serviço deve envolver a satisfação daquele que o utiliza, a satisfação de quem o presta, o presente estudo avaliou a satisfação comparando a resposta de diferentes fontes de dados (usuários e profissionais) sobre um mesmo aspecto da organização do serviço utilizando os conceitos essenciais da APS, sem utilizar diretamente os termos "satisfeito" ou insatisfeito. Cada aspecto foi avaliado individualmente, por cada participante atribuindo uma nota que variava segundo uma escala única (Tipo Likert).

O perfil dos hipertensos cadastrados nas Unidades de Saúde da Família dos municípios de João Pessoa e Campina Grande revelou um predomínio de idosas com baixo nível de escolaridade e níveis pressóricos não controlados em ambos os sexos. Alega-se que a predominância feminina nos serviços de saúde é fruto do reflexo cultural, motivada pela forma de organização dos serviços (horário de atendimento, localização), levando os homens a aderir menos ao tratamento que as mulheres ${ }^{19}$. Embora não pesquisado neste estudo, foi observado que a hipertensão arterial no sexo feminino tem sido associada à inserção das mulheres no mercado de trabalho e ao movimento de urbanização e desenvolvimento tecnológico existente $^{20}$.

O baixo nível de escolaridade observado nos dois municípios pode sugerir maior dificuldade dos usuários em apresentar uma visão crítica sobre o que é um serviço organizado para atender suas necessidades de acompanhamento e adesão. Para um indivíduo que tem um acesso limitado ao serviço de saúde, o simples fato de poder frequentar uma unidade, realizar consultas e receber a medicação necessária remete-o a responder os questionamentos com escores elevados ${ }^{19}$. Aidade, por sua vez, com predominância de pessoas com idade avançada, é citada na literatura como um fator que leva o indivíduo a avaliar bem o serviço, seja pelo maior carisma, seja pela menor expectativa. Todavia, 
alguns estudos apontam que as mulheres estão mais aptas a criticar os serviços, constituindo-se em boas informantes para as pesquisas ${ }^{10}$.

Embora em Campina Grande o número de acompanhados tenha sido ligeiramente maior, ainda foi observado um percentual maior de usuários com níveis pressóricos não controlados, embora em uma proporção menor do que em João Pessoa. Todavia, em outros estudos, não foi observada associação entre o acompanhamento e o controle pressórico ${ }^{19}$. Ressalta-se que o controle pressórico não depende unicamente do acompanhamento pelo serviço. Fazse necessário uma atitude global em relação à própria saúde e exige participação ativa dos hipertensos. Para isso é necessário o comparecimento às consultas e a mensuração regular da pressão arterial a fim de avaliar o controle da hipertensão.

Os usuários hipertensos de Campina Grande mostraram-se mais insatisfeitos do que os usuários de João Pessoa em todas as dimensões, exceto para a dimensão elenco de serviços, havendo um empate. Em cinco das sete dimensões, a comparação entre os dois municípios deixa clara a diferença na percepção de satisfação dos usuários. Com relação à dimensão Coordenação, observouse diferença significativa na satisfação dos usuários de Campina Grande. A Coordenação pressupõe alguma forma de continuidade da atenção, seja por parte do atendimento pelo mesmo profissional, seja por meio de prontuários médicos, ou ambos, além do reconhecimento de problemas abordados em outros serviços e a integração deste cuidado no cuidado global do paciente. A dificuldade em avaliar esta dimensão reside no fato de que a avaliação desse componente envolve a relação da Atenção Primária com outros níveis de atenção ${ }^{5}$.

A Dimensão Enfoque na Família apresentou valores regulares e insatisfatórios para João Pessoa e Campina Grande, respectivamente. A família é o pilar fundamental no tratamento de qualquer doença, principalmente da hipertensão que é um problema crônico e que requer atenção sob todos os seus aspectos $^{11}$. A orientação familiar considera o contexto familiar na atenção integral, chamando a atenção para a necessidade de ações voltadas para esse objetivo ${ }^{21}$.

A Orientação para a Comunidade apresentou valores insatisfatórios para ambos os municípios, apresentando índices piores para o município de
Campina Grande. Essa dimensão envolve o reconhecimento das necessidades sociais por meio da identificação dos problemas de saúde da comunidade através de dados epidemiológicos e do contato direto com a comunidade, a fim de desenvolver ou adequar ações para resolução destes problemas, assim como o planejamento e a avaliação conjunta dos serviços ${ }^{21}$.

O usuário deve ser reconhecido como sujeito detentor de um saber sobre o processo saúdedoença, capaz de estabelecer uma interlocução dialógica com o serviço de saúde e formular uma análise crítica sobre a realidade e o aperfeiçoamento das estratégias na luta cotidiana de controle da hipertensão ${ }^{11}$.

Os profissionais apresentaram-se mais satisfeitos do que os usuários para os dois municípios. Os serviços são mais bem avaliados por quem os prestam e, dentro de quem os prestam ${ }^{5}$. O produto nos serviços de saúde resulta do contato direto entre profissionais e usuários e das ações e interações que resultam dessa relação ${ }^{12}$.

As dimensões Acesso ao Tratamento, Coordenação e Orientação para a Comunidade apresentaram diferenças significativas quando comparadas entre os profissionais dos dois municípios.

A dimensão Acesso ao Tratamento recebeu melhor avaliação dos profissionais de João Pessoa. O acesso tem inter-relação com a resolubilidade e extrapola a dimensão geográfica, abrangendo aspectos de ordem econômica, cultural e funcional de oferta de serviços ${ }^{21}$. Existem muitas dificuldades de acessibilidade e acesso devido à desproporção entre demanda e oferta de serviços. Tal fato pode ser justificado ao verificar uma maior cobertura da ESF para o município de João Pessoa.

A dimensão Coordenação recebeu avaliação satisfatória dos profissionais de João Pessoa com índices regulares para Campina Grande. A coordenação se realiza no cuidado individual exercido pelos profissionais de saúde no processo de atenção que articula diferentes prestações e se concretiza na continuidade da atenção, que deve ser centrada no paciente, adequada às suas necessidades de saúde e oportuna - ofertada em tempo hábil. A coordenação dos cuidados exige a definição e o conhecimento, por parte dos profissionais, das ações desenvolvidas por cada 
serviço que compõe a rede de saúde como elemento do processo de integração entre os diferentes níveis de atenção.

Os grupos dos portadores de doenças crônicas demandam atenção contínua e trabalho preventivo com monitoramento das ações desenvolvidas. Os portadores de doenças crônicas enfrentam grandes dificuldades de acessar o sistema, pois os demais grupos populacionais têm sido tradicionalmente alvo de programas de saúde e reconhece-se a dificuldade para responder, de forma satisfatória, à questão do acesso e à resolutividade do sistema ${ }^{22}$.

A dimensão Orientação para a comunidade apresentou índices regulares para ambos os municípios, apresentando índices mais baixos para Campina Grande. Neste caso, observa-se a necessidade de ações que estimulem uma maior participação dos profissionais dentro da comunidade como apoio resolutivo acerca da doença e seu controle. Devem-se estimular as trocas interpessoais, contrapondo-se à passividade usual das práticas educativas tradicionais ${ }^{11}$.

Ao comparar a satisfação de usuários e profissionais percebe-se que há uma maior satisfação para os profissionais de ambos os municípios. Em João Pessoa observou-se diferença na satisfação entre os atores para as dimensões: Acesso ao Diagnóstico, Acesso ao Tratamento, Adesão/vínculo, Elenco de Serviços, Enfoque na Família e Orientação para a Comunidade. Para Campina Grande a diferença foi ressaltada nas dimensões: Acesso ao Tratamento, Adesão/vínculo, Coordenação, Enfoque na Família e Orientação para a Comunidade.

A avaliação multidimensional é um processo que resulta na identificação de conflitos e na busca de soluções por meio do envolvimento dos diferentes atores. Logo, observa-se uma diferença entre a experiência vivenciada pelos atores envolvidos. Os conflitos entre as perspectivas de profissionais e usuários são decorrentes de divergências entre modelos explanatórios de saúde, podendo ser resolvidos mediante treinamento e educação.

Em vista disso, no caso da diferença entre as experiências de profissionais e usuários sobre as dimensões pode-se indagar se os altos escores atribuídos pelos profissionais são consequência do conhecimento, apenas teórico, que estes têm sobre os atributos da APS. Aqui, caberia investigar a formação profissional desses atores. Será que esses profissionais teriam formação para atuação em Saúde da Família? Deste modo, para melhorar a atenção, seria necessário investir na ESF, principalmente, na qualificação do acesso e na formação profissional, incluindo o reconhecimento dos especialistas na área de APS e a instituição de um programa de educação continuada que alcance todos os profissionais ${ }^{5}$.

\section{CONCLUSÃO}

O presente estudo permitiu avaliar a satisfação com a atenção voltada para o hipertenso da atenção primária comparando as percepções do usuário e do profissional em dois municípios paraibanos. Até o momento não foram encontrados estudos representativos, utilizando esta metodologia, realizados no Brasil e, em particular, no Nordeste, nos quais há maior preocupação por parte do Ministério da Saúde em controlar doenças crônicas não transmissíveis por meio da Estratégia Saúde da Família.

Para ambos os municípios foi possível identificar os níveis de satisfação dos usuários como regulares ou baixos, enquanto os níveis de satisfação dos profissionais apresentaram-se elevados, sugerindo que o cuidado não está sendo capaz de atender às necessidades do usuário, ou estas necessidades não estão sendo compreendidas pelos profissionais.

Deste modo, foi possível levantar questionamentos e gerar subsídios para que os gestores em saúde possam identificar os pontos vulneráveis expressados por meio das dimensões avaliadas e, consequentemente, à falta de controle pressórico, contribuindo para o processo de tomada de decisão, a fim de subsidiar a elaboração e implementação de políticas públicas racionais e de práticas de saúde mais eficientes para o controle da hipertensão arterial.

Sugere-se que os resultados desse estudo possam ser utilizados para fornecer elementos científicos de apoio para outras Unidades de Saúde da Família (USF) e instituições governamentais, através da aplicação dessa metodologia em avaliações dos serviços de saúde aos hipertensos. 


\section{REFERÊNCIAS}

1. Brasil. Avaliação na Atenção Bási [RBCS] Distribuição dos Fonoaudiólogos que atendem ao SUS no Estado da Paraíba, Brasilca em Saúde. Brasília: editora do Ministério da Saúde; 2005.

2. Gouveia GC. Avaliação da satisfação dos usuários com o sistema de saúde brasileiro. [Tese de doutorado].Recife: Centro de Pesquisas Aggeu Magalhães, Fundação Oswaldo Cruz; 2009. 189p.

3. Silva LMV, Formigli VLA. Avaliação em Saúde/ : Limites e Perspectivas. Cad Saúde Pública. 1994;10(1):80-91.

4. Almeida C, Macinko J. Validação de uma metodologia de avaliação rápida das características organizacionais e do desempenho dos serviços de atenção básica do Sistema Único de Saúde (SUS) em nível local. Brasília: Organização Pan-Americana de Saúde; 2006. 1-215 p.

5. Castro RCL, Knauth DR, Harzheim E, Hauser L, Duncan BB. Avaliação da qualidade da atenção primária pelos profissionais de saúde/ : comparação entre diferentes tipos de serviços. Cad Saúde Pública. 2012;28(9):177284.

6. Elias PE, Ferreira CW, Cecília M, Alves G, Cohn A, Junior ÁE. Atenção Básica em Saúde/ : comparação entre PSF e UBS por estrato de exclusão social no município de São Paulo. Cien Saude Colet. 2006;11(3):633-41.

7. Jorge M, Guimarães J, Vieira L, Paiva F, Rocha e Silva D, Pinto A. Avaliação da qualidade do Programa Saúde da Família no Ceará: A satisfação dos Usuários. Rev Baiana de Saúde Pública. 2007;258-68.

8. Macinko J, Almeida C, de Sá PK. A rapid assessment methodology for the evaluation of primary care organization and performance in Brazil. Health Policy Plan [Internet]. 2007 May [cited 2014 Jan 10];22(3):16777. Available from: http://www.ncbi.nlm.nih.gov/ pubmed/17400576

9. Moraes PA, Bertolozzi MR, Hino P. Percepções sobre necessidades de saúde de um serviço de saúde. Rev Esc Enferm USP. 2011;45(1):19-25.

10. Esperidião MA, Trad LAB. Avaliação de satisfação de usuários/ : considerações teórico-conceituais. Cad Saúde Pública. 2006;22(6):1267-76.

11. Lima VV. Influência da satisfação no controle da pressão arterial sistêmica na Atenção Primária: Análise com modelagem de equações estruturais e indicadores compostos. [Dissertação de Mestrado]. João Pessoa: Universidade Federal da Paraíba; 2013. 112p.

12. Serapioni M. Avaliação da qualidade em saúde. Reflexões teórico-metodológicas para uma abordagem multidimensional. Rev Crit Cienc Sociais. 2009;85:6582.
13. Paes NA. Avaliação da efetividade no controle da Hipertensão Arterial Sistêmica e associação com fatores de risco. Relatório final. Brasília: Ministério da Saúde; 2008.

14. Paes NA. Desempenho do Programa Saúde da Família comparado com o das Unidades Básicas de Saúde no controle da Hipertensão Arterial Sistêmica e fatores associados em municípios do Estado da Paraíba. Relatório final. Brasília: Ministério da Saúde; 2009.

15. Shi L, Starfield B, Xu J. Validating the Adult Primary Care Assessment Tool. J Fam Pract. 2001;50(2):161-75.

16. Savassi LCM. Qualidade em serviços públicos/ : os desafios da atenção primária. Rev bras med fam comunidade. 2012;7(23):69-74.

17. Paes NA, Silva CS, Figueiredo TMRM, Cardoso MAA, Lima JO. Satisfação dos usuários hipertensos com os serviços da rede de Atenção Primária do Brasil. Pan Am J Public Heal. 2014;36(2):87-93.

18. Sociedade Brasileira de Hipertensão. VI Diretrizes Brasileiras de Hipertensão. 2010;13(1):1-66.

19. Silva CS, Paes NA, Figueiredo TMRM, Cardoso MAAC, Silva ATMC, Araujo JSS. Controle pressórico e adesão/ vínculo em hipertensos usuários da Atenção Primária a Saúde. Rev da Esc Enferm da USP. 2013; 47(3):58490

20. Carrijo RL, Car MR. Dialética do modo de vida de portadores de hipertensão arterial/ : o objetivo e subjetivo. Rev Esc Enferm USP. 2007;41(4):573-80.

21. Starfield B. Atenção Primária: equilíbrio entre necessidades de saúde, serviços e tecnologia. Brasília: UNESCO/ Ministério da Saúde; 2002. 1-726 p.

22. Giovanella L, Escorel S, Mendonça MHM, coordenadores. Estudos de caso sobre a implementação da Estratégia Saúde da Família em grandes centros urbanos. Relatório final. Brasília: Ministério da Saúde; 2009.

\section{Correspondência}

Christiana Souto Silva

R Cel. Souza Lemos, 307, Miramar.

CEP: $58043-190$

João Pessoa - PB, Brasil.

christianasouto@yahoo.com.br 